Revue pluridisciplinaire du monde antique

$19 \mid 2003$

Le statut et l'image du corps dans la mythologie et la littérature grecques (suite et fin)

\title{
Corps des citoyens, corps de la cité
}

\section{Michelle Lacore}

\section{(2) OpenEdition}

\section{Journals}

Édition électronique

URL : https://journals.openedition.org/kentron/1858

DOI : $10.4000 /$ kentron. 1858

ISSN : 2264-1459

\section{Éditeur}

Presses universitaires de Caen

\section{Édition imprimée}

Date de publication : 31 décembre 2003

Pagination : 143-158

ISBN : 2-84133-222-5

ISSN : 0765-0590

\section{Référence électronique}

Michelle Lacore, "Corps des citoyens, corps de la cité », Kentron [En ligne], 19 | 2003, mis en ligne le 12 avril 2018, consulté le 13 octobre 2022. URL : http://journals.openedition.org/kentron/1858 ; DOI :

https://doi.org/10.4000/kentron.1858

\section{(c) (i) (9)}

Creative Commons - Attribution - Pas d'Utilisation Commerciale - Pas de Modification 4.0 International - CC BY-NC-ND 4.0

https://creativecommons.org/licenses/by-nc-nd/4.0/ 


\section{CORPS DES CITOYENS, CORPS DE LA CITÉ}

L'emploi métaphorique du mot « corps» pour représenter la communauté des citoyens est parfaitement usuel dans la réflexion politique moderne. Or, paradoxalement, en dépit du rôle fondateur de la pensée politique des Grecs dans la réflexion occidentale, de la Renaissance à l'époque moderne ${ }^{1}$, la métaphore même du corps politique et de ses membres ne plonge pas ses racines dans la réflexion politique de l'époque classique grecque (celle des v et $\mathrm{v}^{\mathrm{e}}$ siècles). Elle est pourtant attestée dans la réflexion de l'époque hellénistique et romaine et remonte peut-être à de très anciennes origines proche-orientales. Cependant l'origine de l'omniprésence de cette métaphore dans la pensée occidentale nous semble être essentiellement chrétienne, à partir du Nouveau Testament et des écrits des Pères de l’Église.

\section{Le corps de la cité dans la pensée classique : une métaphore problématique}

L'usage commun de l'époque classique reflète la perception de l'individu humain comme une totalité, ce qui explique par exemple l'emploi universel par orateurs et historiens de sôma pour désigner non pas le corps seul, mais l'individu, la personne, dans sa totalité et sa singularité. Cependant, on ne relève pas, dans cette perspective, d'emploi métaphorique vraiment significatif du terme « corps » passant du corps des citoyens (c'est-à-dire de leur personne) à la cité définie comme un corps abstrait constitué par la somme des corps ou des personnes des citoyens, ni chez les orateurs ou les historiens, ni chez les philosophes.

À l'aide du TLG - E et du programme « complex search» de Pandora nous avons cherché en effet les occurrences dans la prose classique des associations entre "corps», «membres» et "cité», "citoyens », qui pourraient véhiculer cette métaphore. Les résultats d'une telle recherche sont extrêmement maigres : ni Hérodote, ni Thucydide, ni Xénophon, ni Lysias, ni Antiphon, ni Andocide, Isée, Eschine ou Lycurgue ne présentent d'exemples de cette figure de style. Les rarissimes occurrences de l'image du corps de la cité chez les orateurs sont d'une faiblesse remarquable, et le bilan des

1. Voir par exemple l'histoire de la redécouverte de la Politique d'Aristote à la fin du Moyen Âge et de son influence sur la réflexion politique en Occident dans Aubonnet ${ }^{2}$ 1991, CXLVI-CXCVI. 
philosophes - Aristote aussi bien que Platon - pour des raisons très différentes, est lui aussi négatif.

\section{Chez les orateurs}

Isocrate présente, il est vrai, un exemple de cette métaphore mais l'image des citoyens constituant un corps n'apparaît ici que de façon indirecte et dévalorisante. Ainsi l'orateur, au moment de proposer une réforme constitutionnelle restaurant les pouvoirs anciens du conseil aristocratique de l'Aréopage, donne cette définition célèbre de la constitution dans le discours Aréopagitique (14), définition qu'il a répétée mot pour mot dans son ultime discours, le Panathénaïque (138):

«L'âme de la cité n'est rien d'autre que la constitution, qui a le même pouvoir que dans le corps la pensée : c'est elle qui délibère sur tout...»

Il est certain que le texte suggère implicitement que l'ensemble des citoyens constitue le corps de la cité, mais la métaphore n'a pour fonction que d'affirmer l'incapacité, l'infériorité de ce «corps » par rapport à l'élément dirigeant, la constitution,

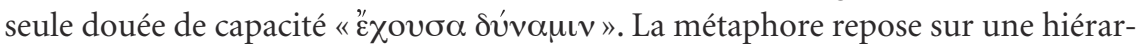
chie des constituants de l'être humain et donc infériorise le corps des citoyens. Si les citoyens constituent un corps, ce n'est que dans la mesure où ce corps a besoin d'âme pour pleinement exister. Il n'est nullement question de la solidarité des citoyens comme membres d'un même corps, mais seulement du pouvoir d'animation et de direction de la constitution.

Alors que la personnification de la cité est particulièrement vibrante chez Démosthène ${ }^{2}$, la métaphore du corps de la cité apparaît plus inexistante encore chez ce patriote, démocrate « radical». L'idée de la cité comme corps en ce qu'elle ne peut se passer d'une âme, n'apparaît que dans un fragment très incertain, dans un recueil de «bons mots » qu'Aristote aurait constitué:

Démosthène l'orateur disait que les lois sont l'âme de la cité: de même que le corps privé d'âme succombe, de même une cité, faute de lois, va à sa ruine ${ }^{3}$.

Le remplacement de la «constitution» par les «lois» comme âme de la cité est cependant significatif, dans la mesure où les lois, sans cesse en devenir, sont la création

2. Est-il besoin de mentionner le Discours sur la Couronne, 170, où Démosthène évoque la patrie appelant, dans le silence de l'assemblée devant la catastrophe de la prise d'Élatée, l'intervention d'un citoyen capable d'assurer son salut?

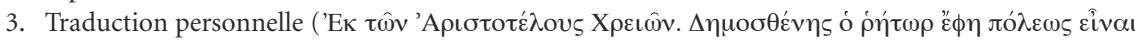

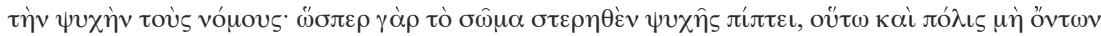

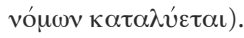


continue de l'assemblée des citoyens. Par ailleurs la conception de l'âme proposée est moins idéaliste que celle d'Isocrate : l'âme apparaît moins ici comme un principe d'autorité que comme le souffle de la vie, ce qui est une des valeurs de psyché encore à l'époque classique. La métaphore du corps de la cité est ainsi porteuse de l'idée de mortalité doublement soulignée dans la phrase.

La dernière pièce de ce dossier concernant les orateurs se trouve dans un discours fragmentaire d'Hypéride Contre Démosthène, à l'occasion de la sombre affaire du détournement de fonds appartenant au macédonien Harpale et saisis par Athènes; Hypéride déclare que, non contents de la bénignité de la cité qui avait toujours permis aux stratèges et aux politiciens de réaliser de substantiels profits, pourvu que ce ne fût pas à son détriment, Démosthène et Démade «ont accepté des cadeaux

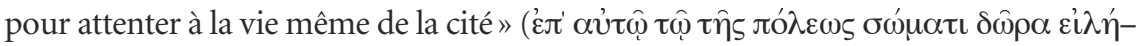
$\phi \alpha \sigma \iota)^{4}$, littéralement «visant le corps même de la cité». La formule est obscure, l'affaire d'Harpale demeure d'ailleurs opaque aux yeux des historiens modernes ${ }^{5}$. La mention du « corps de la cité» semble surtout un procédé oratoire destiné à souligner la gravité de l'atteinte portée aux intérêts matériels et diplomatiques d'Athènes par ces prélèvements malhonnêtes opérés sur le trésor d'Harpale, que réclame la Macédoine.

Si la métaphore du corps de la cité n'a qu'une existence improbable et inconsistante chez les orateurs, après avoir été totalement ignorée des historiens des $\mathrm{v}^{\mathrm{e}}$ et Iv ${ }^{e}$ siècles, les philosophes semblent la bannir, rencontre que la divergence des positions d'Aristote et de Platon rend d'autant plus remarquable.

\section{Chez Platon : le corps moins réel que l'âme}

Il y a bien un recours isolé chez Platon, non pas à la métaphore du corps de la cité, mais à une comparaison entre la cité bien administrée et un corps affecté solidairement dans toutes ses parties par les mêmes impressions de plaisir et de peine. Cependant cette unité de sentiments ne concerne que la classe des gardiens et résulte de la communauté des femmes et des enfants entre gardiens. D'autre part Platon oscille, pour définir cette unité, entre la mention de l'individu tout entier, corps et

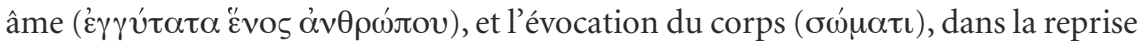
de la comparaison ${ }^{6}$.

Dans tout le reste de son œuvre, en revanche, c'est l'âme qui est le point de référence de la réflexion sur la cité. Dès le Gorgias, Socrate définit la politique comme

4. Hypéride, Contre Démosthène (Colin 1946).

5. Colin 1946, notice p. 228-229 avoue sans ambages son embarras et se refuse à admettre que Démosthène ait agi par pure cupidité.

6. Platon, République, V (Chambry 1933), 462c-d et 464b. Le principe de la communauté des femmes, des enfants et des biens est vivement critiqué par Aristote, Politique, II, 1262a-b. 
« un art de l'âme». Face au sophiste Pôlos, Socrate, par une comparaison complexe, met en rapport les arts véritables qui se rapportent au corps et à l'âme :

«L'art qui se rapporte à l'âme, je l'appelle la politique; pour celui qui se rapporte au corps $[\ldots]$ je distingue deux parties, la gymnastique et la médecine. Dans la politique, je distingue la législation, qui correspond à la gymnastique, et la justice ${ }^{7}$, qui correspond à la médecine...»

Le philosophe, par un glissement hardi, mais implicite, passe du citoyen individuel à la cité, mais cette comparaison complexe entre les arts de l'âme et ceux du corps a pour résultat que la métaphore médicale se trouve idéalisée, rapportée à la cité comme âme et non comme corps: la législation prévient et la justice soigne les maladies non pas du corps, mais de l'âme de la cité.

Dans l'ensemble de la République, l'orientation idéaliste se trouve amplifiée. Le point de vue n'est plus celui du médecin, comme dans le Gorgias, mais du géomètre, bâtissant une cité dans l'ordre des esprits. Ce n'est pas sur la métaphore du corps que Socrate s'appuie, mais sur celle de l'âme.

Sommé de définir la vertu de justice dans l'âme individuelle, Socrate déclare ne pouvoir le faire qu'en raisonnant à plus grande échelle (en grandes lettres ${ }^{8}$ ) sur la justice dans la cité. Cette fois, le glissement de l'individu à la collectivité est ouvertement revendiqué. La cité juste est une âme dans laquelle les différentes composantes remplissent, sans empiètement, les fonctions qui leur sont propres: les philosophes dirigent la cité, les gardiens assurent sa défense, les producteurs subviennent à ses besoins, hiérarchie qui reflète celle de l'âme, dans laquelle le plus noble composant est le nous (l'esprit), capable de maîtriser et réguler l'ardeur du thymos (la colère, le courage) et l'entraînement des epithumiai, les passions, les désirs. C'est donc l'image d'une âme où les différentes fonctions sont bien équilibrées et hiérarchisées, non celle d'un corps, qui figure la cité.

Le rejet de cette métaphore du « corps de la cité» est même, à notre avis, fondamental dans le texte de Platon et lié au mépris dans lequel sont tenus le corps et l'ordre de réalités auquel il appartient. C'est là un thème bien connu de la pensée platonicienne et il suffit peut-être de rappeler une simple formule présente en deux passages du début et de la fin de son œuvre: «l'homme n'est rien d'autre que son

7. Platon, Gorgias, 464b (Croiset 1923). Le texte du Gorgias comporte une difficulté qui disparait dans la traduction en français en raison de la polysémie du terme « justice». Platon distingue habituellement la justice comme institution ou comme entité morale c'est-à-dire dík $\eta$, de la justice comme

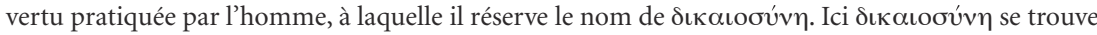
inexplicablement employé et a toutes les chances d'être la leçon authentique, puisqu'il semble attesté dans le Gorgias à l'époque alexandrine, voir la note de Dodds 1969, 228.

8. Platon, République, II, 368d. 
âme ${ }^{9}$. Cette formule est même rappelée par Aristote dans un passage de la Politi$q u e^{10}$ où il met au jour une contradiction entre ce postulat et les étapes de développement de la cité tel que Socrate se le représente.

\section{Aristote: le refus du totalitarisme}

On pourrait s'attendre en passant de Platon à Aristote, très attaché, lui, aux sciences de la vie et à l'expérience, à ce que la métaphore du corps des citoyens occupe une place importante dans ses divers traités touchant à la politique.

Il n'en est rien, en dépit de ce que pourraient laisser croire les expressions utilisées parfois par les spécialistes d'Aristote. Ainsi l'auteur d'un éminent traité consacré à la pensée politique de Platon et d'Aristote voit-il chez le second « une assimilation absolue de l'État au corps humain et de ses citoyens aux organes du corps » ${ }^{11}$. C'est aussi l'impression que donne la lecture rapide de la traduction française de la Politique d'Aristote qui figure dans l'édition de référence ${ }^{12}$. En raison de l'inexistence du neutre dans la langue française, du goût de notre langue pour les substantifs précis et, il faut bien le dire, en raison aussi de nos habitudes de pensée, le terme de "corps » de citoyens ou de juges apparaît à d'innombrables reprises dans la traduction, de même que le terme de «membres» de ces "corps", sans que l'on trouve une seule fois à ces emplacements dans le texte grec sôma ou mélè ${ }^{13}$. L'index verborum de J. Aubonnet avoue d'ailleurs la présence d'un seul exemple de $\tau \grave{\alpha} \mu \mu^{\prime} \lambda \eta$ au sens de «membra» en 1336a 10, texte relatif à des problèmes d'orthopédie. Tous les autres exemples de $\mu \dot{\varepsilon} \lambda \mathrm{o} / \mu \dot{\varepsilon} \lambda \eta$ ont, selon le même index, la valeur de compositio musica. Pourtant l'index général qui lui fait suite, à la rubrique " corps » et à la rubrique "membres ", laisse croire à de nombreux exemples de la métaphore chez Aristote.

Cette traduction, trop littéraire et pas assez littérale, gomme l'abstraction bien connue du vocabulaire d'Aristote, pourtant particulièrement intéressante pour notre propos. On trouve plusieurs termes abstraits désignant la collectivité đò ö $\lambda$ ov le

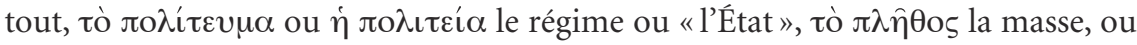

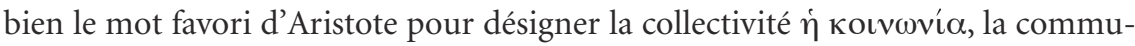
nauté. Ce que le traducteur rend par «être membre» correspond à l'idée d'«avoir

9. Platon, Alcibiade, 130c, Lois, XII, 959b.

10. Aristote, Politique (Aubonnet ${ }^{2}$ 1989), 1291a, 1. 24 (IV, 4, 14). "Si donc on admet que l'âme est une partie de l'être vivant plus réellement que ne l'est le corps...»

11. Barker 1959, 277: "an absolute assimilation of the State to the human body and of its citizens to the bodily organs."

12. Aristote, Politique (Aubonnet $\left.{ }^{2}, 1986-1991\right)$.

13. Un exemple, entre des dizaines: Aristote, Politique (Aubonnet $\left.{ }^{2} 1989\right), 1299 \mathrm{~b}(\mathrm{IV}, 15,11)$ : $\beta$ ou $\lambda \dot{\eta} \delta \dot{\varepsilon}$

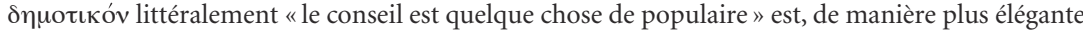
mais trompeuse, surtout en raison de la répétition du procédé, rendu par «le conseil, lui, est un corps populaire». 
part à» ( $\mu \varepsilon \tau \dot{\varepsilon} \chi \varepsilon\llcorner v)$, le terme «membre» traduit souvent un terme aristotélicien rigou-

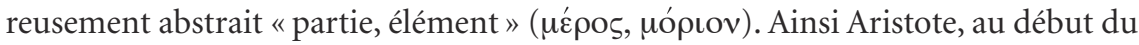
livre III de la Politique, définit-il la cité comme un tout qui est une pluralité:

"Comme la cité fait partie des composés au même titre que n’importe quel autre "tout" composé de plusieurs éléments, il est clair que notre recherche doit porter d'abord sur le citoyen; la cité, en effet, est une collectivité de citoyens ${ }^{14}$.

Lorsque le «corps» fournit un point d'appui aux analyses d'Aristote, ce n'est jamais sous la forme de l'assimilation de la cité à un organisme vivant qu'implique la métaphore. La notion de «corps » apparaît réduite à une extrême abstraction, presque fossilisée, dans les premières pages de la Politique, au moment où Aristote, qui a défini l'être humain comme un " animal politique», veut par une image illustrer la préexistence du tout par rapport aux parties:

«Par nature donc, la cité est antérieure à la famille et à chacun de nous, car le tout

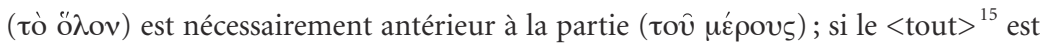

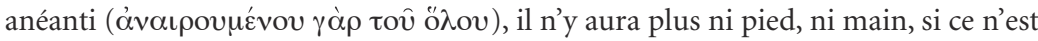
par une analogie verbale, comme on dit une main de pierre: telle sera en effet la main une fois morte $»^{16}$.

On ne trouve ici aucune esquisse de métaphore, seulement une mise en parallèle dans laquelle le corps - qui n'est pas nommé - est envisagé de façon presque mécaniste, en pièces détachées.

En revanche, le corps est mentionné lorsque le philosophe définit la cité comme un être composite, au même titre que n'importe quel être vivant, composé d'une âme et d'un corps, mais il s'agit seulement de souligner l'hétérogénéité des espèces

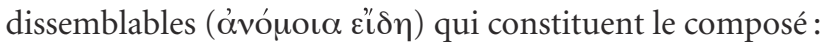

«La cité se compose d'éléments dissemblables - de même qu'un être vivant, à titre de premier exemple, se compose d'une âme et d'un corps, une âme de raison et de désir, une famille d'un homme et d'une femme [...] de la même manière la cité se compose de tous ces éléments et d'autres qui sont dissemblables... ${ }^{17}$

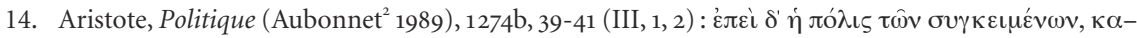

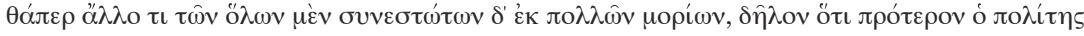

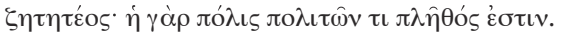

15. J. Aubonnet croit ici utile de rétablir « le corps », mais c'est précisément l'absence du mot qui est, à notre avis, digne d'intérêt.

16. Aristote, Politique (Aubonnet ${ }^{2}$ 1991), 1253a, 20-23 (I, 2, 13).

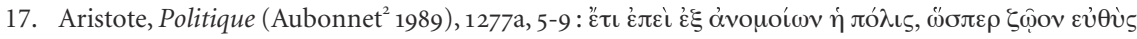

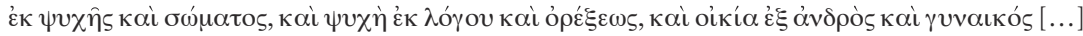

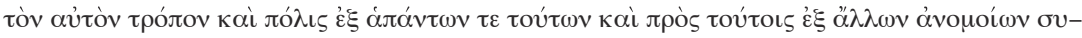

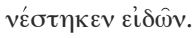


Le corps fournit parfois des images monstrueuses. Ainsi en est-il lorsqu'Aristote explique les altérations que subissent les régimes politiques par une anomalie de croissance analogue à celle qui peut s'observer dans le corps :

« Des changements de régime ont aussi pour cause un accroissement disproportionné. De même en effet que le corps se compose de parties et que sa croissance doit s'opérer suivant leurs mutuelles proportions pour que demeure l'harmonie d'ensemble, faute de quoi elle est détruite [...], de même également une cité se compose de parties, dont l'une quelconque s'accroît souvent sans qu'on s'en aperçoive: la masse des pauvres dans les démocraties et les polities ${ }^{18}$.

C'est bien l'image de la croissance du corps qui est utilisée pour penser la croissance défectueuse de la cité; toutefois il ne s'agit pas d'une métaphore, mais bien d'une comparaison, presque lourdement articulée, maintenant la distance entre les deux termes, distance que la métaphore abolirait.

On peut encore citer en ce sens un passage très intéressant du livre III où Aristote veut illustrer la supériorité du tout sur ses parties ${ }^{19}$ :

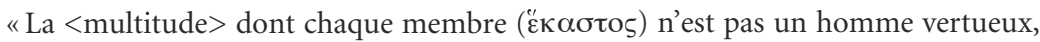
peut cependant, par l'union de tous être meilleure que cette élite, non pas individuellement, mais collectivement [...]. Du fait qu'ils sont plusieurs, chacun a sa part de vertu et de sagesse pratique et, de leur union, naît comme un seul homme à plusieurs pieds, plusieurs mains et doué de plusieurs sens et il en va de même pour le caractère et l'intelligence. Voilà pourquoi la multitude est le meilleur juge des œuvres de la musique et de celles des poètes: les uns apprécient une partie, les autres une autre et tous apprécient le tout.»

Dans ce contexte qui prône sans ambiguïté les valeurs de la démocratie, Aristote recourt à l'image d'un corps monstrueux résultant de l'addition de tous les corps individuels qui le composent, image qui n'est pas sans évoquer celle de la bête polycéphale qui représente la partie instinctive de l'âme en République, IX, 588c-e. Utiliser l'image du corps en ce sens monstrueux, c'est suggérer son inadéquation pour exprimer la pluralité des individus, à laquelle Aristote est attaché.

En effet le refus de la métaphore du corps ne s'explique pas chez Aristote par le rejet ou le mépris du corps. Le corps n'est pas nié, qu’il soit conçu (suivant les œuvres d'Aristote auxquelles on s'attache) comme instrument de l'âme ou matière dont

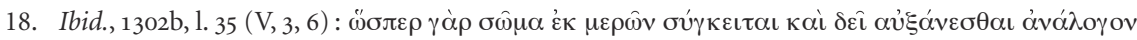

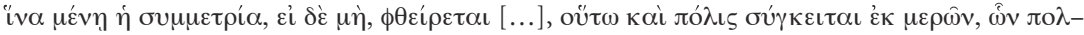

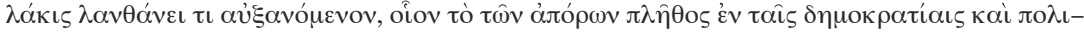

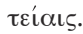

19. Ibid., 1281b, 1. 7 (III, 11, 2). 
elle est la forme ; au contraire il joue un rôle très important dans la définition aristotélicienne de l'individu, comme le note D. J. Allan ${ }^{20}$.

Que l'unité organique du corps ne puisse être utilisée pour représenter celle de la cité participe, selon nous, du refus du totalitarisme, mis en lumière par M. CantoSperber ${ }^{21}$, qui évoque les critiques adressées par Aristote à la cité platonicienne, dans laquelle une unité trop poussée détruit la nature propre de la cité. L'auteur relève les nombreuses affirmations aristotéliciennes du fait que l'unité de la cité est une unité de composition, une symphonie qui ne saurait être ramenée à l'unisson, avec cette critique déclarée des excès de l'unité de la cité platonicienne:

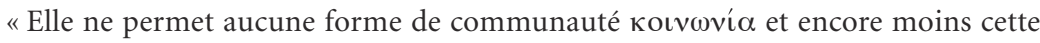
forme de communauté des sentiments qu'est l'amitié ( $\phi \iota \lambda i ́ \alpha)$ essentielle à la vie politique.»

C'est bien aussi dans le sens d'un profond attachement à la démocratie que P. Aubenque ${ }^{22}$ interprète les analyses d'Aristote et il définit de façon éclairante la koinônia si chère à Aristote :

«Le "gouvernement du milieu" qu’on l'appelle "politie" ou "démocratie" est la plus excellente des constitutions [...] parce qu'il ouvre et maintient un espace, un "milieu" de la parole et des biens échangés [...], des aspirations communes...»

Nous aimerions ajouter qu'Aristote, tout en affirmant la distinction des constituants de la cité ainsi que leur diversité, jugée par lui positive, souligne l'égalité des parties dissemblables dans le meilleur des régimes, égalité qui est assurée par la permutabilité des deux rôles complémentaires, commander et obéir, que doivent assurer à tour de rôle - non seulement par l'effet de l'âge, mais par la rotation des fonctions électives - les citoyens. Il s'agit même d'un véritable leitmotiv:

«Le bon citoyen doit savoir et pouvoir obéir et commander ${ }^{23}$.

C'est, à nos yeux, l'attachement à l'individualité du citoyen et à l'égalité entre les citoyens qui explique l'absence chez Aristote de la métaphore du corps de la cité. Aristote, loin d'être un pur théoricien, est tout nourri de la tradition politique classique:

20. Allan 1965, 57 .

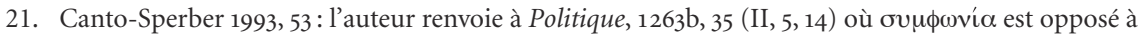

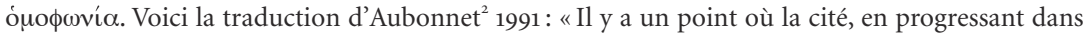
l'unité, cessera d'en être une, et un autre où elle sera encore une cité, mais [...] une cité inférieure; comme si on faisait de la symphonie un unisson, ou du rhythme une unique mesure. »

22. Aubenque 1993, 264: l'auteur renvoie à Politique, 1282a, 19-23 (III, 11, 14), qu'il considère comme «le renversement le plus décidé et le plus conséquent de la thèse antidémocratique de Platon ».

23. Aristote, Politique (Aubonnet ${ }^{2}$ 1989), 1277b, 14 (III, 4, 15). 
l'élaboration de sa Politique a été précédée d'un intense travail de documentation et d'analyse consacré aux constitutions du monde grec et à leur histoire ${ }^{24}$.

En revanche, les emplois de l'image du corps de la cité, postérieurs à l'époque classique, que nous allons maintenant envisager font apparaitre une connotation fondamentalement anti-égalitaire attachée à cette métaphore.

\section{La métaphore d'un ordre fondé sur l'inégalité}

\section{La fable de Ménénius Agrippa}

Nous allons nous permettre une incursion dans le domaine de l'histoire romaine, sans vraiment quitter le domaine grec cependant.

L'apologue du conflit entre les membres et l'estomac, par lequel le "plébéien » Ménénius Agrippa, émissaire des sénateurs, réussit à convaincre la plèbe romaine de mettre fin à sa sécession en l'an 494 avant J.-C. n'est attesté que bien postérieurement à l'époque classique grecque : la fable est rapportée de manière à peu près contemporaine par Denys d'Halicarnasse ${ }^{25}$ et Tite-Live ${ }^{26}$ - qui sera notre seul témoin latin - dans le dernier tiers du $\mathrm{I}^{\mathrm{er}}$ siècle avant J.-C., avant d'être reprise, au tournant du i ${ }^{\text {er }}$ siècle après J.-C., par Plutarque ${ }^{27}$, puis cent ans plus tard par Dion Cassius, cité par le compilateur byzantin Zonaras ${ }^{28}$. La révolte et la « grève» des membres, las de travailler pour assouvir l'appétit insatiable de l'estomac, mènent le corps tout entier et chaque membre en particulier à la décrépitude mortelle, démontrant l'utilité de l'estomac qui répartit par la digestion la nourriture dans tout le corps. Les «membres» sont d'ailleurs entendus en un sens assez lâche puisque sont énumérées les mains, la bouche, les dents, et cette approximation est une constante remarquable de tous les textes païens ou chrétiens qui utilisent la métaphore. Tite-Live, qui a rapporté au discours indirect l'apologue, ne fait qu'esquisser en une phrase de récit l'application de la fable à la sécession de la plèbe hostile aux patriciens ${ }^{29}$ :

« En montrant combien cette sédition intestine du corps ressemblait à la colère de la plèbe contre les patriciens, Agrippa rendit les esprits traitables $»^{30}$.

24. Voir Aubonnet ${ }^{2}$ 1991, LXXXII-XCVI ou, pour les préoccupations historiques d'Aristote dans la Politique, Weil 1960.

25. Denys d'Halicarnasse, Antiquités romaines, VI, 86.

26. Tite-Live, Histoire romaine (Lasserre 1950), II, 32, 8 sq.

27. Plutarque, Vie de Coriolan, 6.

28. Cassius Dio, Historiae Romanae, 7, 14, 6-14.

29. Ogilvie 1965, 313, voit là une manière de contourner la difficulté de donner la parole au "plébéien » Ménénius Agrippa et de préserver la vraisemblance et la tenue du récit historique.

30. Tite-Live, Histoire romaine (Lasserre 1950), II, 32, 12. 
Denys d'Halicarnasse donne au discours un développement extraordinaire, tout nourri de la tradition grecque relative aux dissensions et aux réconciliations, et ne fait intervenir l'apologue qu'à l'extrême fin, comme ultime argument, en sou-

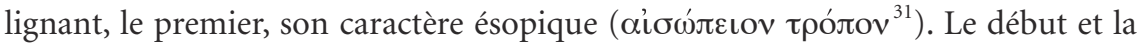
fin de l'apologue sont très intéressants. L'orateur expose d'emblée la métaphore du corps : «il y a une certaine ressemblance entre une cité et un corps humain » ${ }^{32}$. La conclusion met l'accent - et Denys est suivi en cela par Plutarque - sur le rôle irremplaçable et prééminent du sénat:

Ainsi dans les cités, le sénat ${ }^{33}$ qui administre les affaires communes et prévoit ce qui convient à chacun, préserve, garde et corrige toute chose...

Le commentaire de Dion Cassius envisage toute l'affaire en termes de conflit entre riches et pauvres et sa "morale » présente de très intéressants échos des idées conservatrices modérées d'Isocrate ${ }^{34}$ :

Ce discours fit comprendre au peuple que la fortune des gens aisés est aussi un bienfait pour les démunis et que, si les premiers tirent profit de leurs créances et accroissent leur fortune, cela n'est pas au désavantage de la multitude, puisque si les riches étaient dépourvus de biens, les pauvres n'auraient pas, même dans les temps de nécessité, à qui emprunter et périraient sous le poids du dénuement.

Les commentateurs modernes donnent raison à Denys d'Halicarnasse pour ce qui est des origines grecques de la fable ${ }^{35}$, remontant jusqu'à la figure mythique d'Ésope (situé par la tradition au vi siècle avant J.-C. en plein archaïsme grec, mais les textes mis sous son nom n'ont été rassemblés qu'à l'époque impériale romaine). B.E. Perry fait figurer dans ses Aesopica ${ }^{36}$ le texte même de Tite-Live et par ailleurs le corpus ésopique de la Collectio Augustana (de date très tardive, fin de l'Antiquité)

31. Denys d'Halicarnasse, Antiquités romaines, VI, 83, 2. Plutarque et Dion Cassius parlent simplement de «fable» $(\mu \hat{v} \theta$ os $)$.

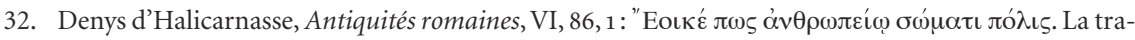
duction des différents extraits de Denys et de Dion Cassius est personnelle.

33. Le conseil ( $\beta$ $v \lambda \eta \dot{)}$ dont il est question ici est bien l'institution aristocratique romaine du sénat.

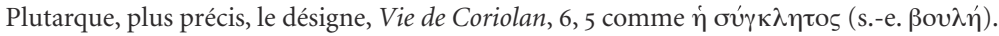

34. Isocrate, Aréopagitique, 31-32 (Mathieu 1942), évoque l'admirable concorde sociale qui régnait entre riches et pauvres au temps où l'Aréopage exerçait sa pleine influence: « Les plus pauvres parmi les citoyens, bien loin de jalouser les possesseurs d'une plus grande fortune, avaient la même sollicitude pour les grandes maisons que pour les leurs propres, parce qu'ils jugeaient que la prospérité de celles-là était une ressource pour eux. "

35. Ogilvie 1965, 312, place l'introduction de cette fable au début de l'historiographie romaine, ce serait l'œuvre de la génération de Fabius Pictor.

36. Perry 1952, fab. 130. 
comporte une ébauche en prose, très fruste, du même motif, intitulée «L'estomac et les jambes ${ }^{37}$.

Toutefois, en dépit du caractère tardif des témoins, cette fable pourrait avoir une origine plus ancienne encore que l'archaïsme grec: M.L. West ${ }^{38}$ évoque une possible source égyptienne de la XXII dynastie (950-730) : un apologue évoquant une «contestation entre l'estomac et la tête » qui serait entré dans la tradition ésopique et s'y serait développé.

Que la fable remonte à l'époque des pharaons ou bien à celle de l'archaïsme grec, elle est de toute façon étrangère à la tradition démocratique : un commentateur récent ${ }^{39} \mathrm{y}$ voit une fable agonistique dans laquelle la concorde - but explicitement assigné dans le récit de Tite-Live à la mission de Ménénius Agrippa ${ }^{40}$ - est finalement assurée par le rétablissement de la hiérarchie de classes contestée : les situations de pouvoir et d'obéissance sont confirmées par la «morale» qui réaffirme la domination de l'estomac sur les membres du corps à son service. On voit en tout cas combien on est loin de la perspective aristotélicienne affirmant la double capacité de chaque citoyen à commander et à obéir.

\section{La métaphore dans la théologie chrétienne : l'Église corps du Christ}

Certains textes du Nouveau Testament présentent, dans leur définition de l'Église, communauté des croyants, des proximités frappantes avec les analyses précédentes. Ces affinités sont d'autant plus intéressantes pour nous que la communauté ecclésiale a, vis-à-vis des individus qui la composent, une vocation englobante comparable à celle de la cité et qu'elle est même dénommée la "Cité de Dieu », par un déplacement de l'expression de l'Ancien Testament qui désignait Jérusalem. L'Église devient la «Jérusalem céleste», la «Cité de Dieu », dès le livre de l'Apocalypse ${ }^{41}$, avec toute la postérité patristique que l'on sait. À la différence de la cité au sens historique classique du terme, qui ne se laissait pas appréhender par la métaphore du corps, cette « cité » d'un nouveau genre trouve là sa représentation privilégiée.

37. Corpus fabularum Aesopicarum (Hausrath and Hunger 1959), fab. 132. Le thème est repris dans une autre fable du recueil ésopique, située dans un ensemble byzantin attribué au philosophe Syntipas

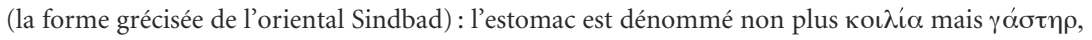
la fable comporte une « morale» de discipline militaire (ibid., Fabulae Syntipae philosophi, fab. 35).

38. West 1969, 119.

39. Zafiropoulos 2001, 82 (pour l'importance de la réciprocité à l'époque archaïque) et 101 (pour le commentaire de la fable Perry 130, c'est-à-dire l'apologue de Tite-Live).

40. Tite-Live, Histoire romaine (Lasserre 1950) II, 32, 7: «Assurément, estimaient les patriciens, il ne restait d'espoir que dans la concorde des citoyens : par tous les moyens, justes ou injustes, il fallait la rétablir dans la cité.»

41. Jean, Ap., 3, 12, 4 . 
On doit toutefois noter une transformation importante dans le registre de la métaphore : J. Schneider a légitimement attiré notre attention sur le caractère cynique de la métaphore «ésopique». Il est vrai que la voracité de l'estomac ne donne pas une image noble des détenteurs du pouvoir, dont le rôle, dans le corps social ou politique, est assimilé au sien dans le corps biologique. D'ailleurs, si l'on admet, pour l'apologue, l'origine égyptienne proposée par M.L. West, la suprématie reconnue à l'estomac dans la version «ésopique » représente un gauchissement de l'original, qui opposait précisément la tête et l'estomac.

La métaphore chrétienne, contrairement aux avatars grecs et romains de l'apologue oriental, met en lumière la noblesse intrinsèque du détenteur de l'autorité, ruinant par là même toute idée de contestation. L'élément dominant l'ensemble du corps est cette fois la tête, c'est-à-dire le Christ. Il s'agit ici exclusivement de textes attribués à l'apôtre Paul, particulièrement soucieux de donner à la communauté des croyants une forme institutionnelle durable, appuyée sur une unité mystique. L'Église forme un seul corps, dont le Christ est la tête ${ }^{42}$. L'unité de ce corps est indivisible, avec une expression défiant la logique pour suggérer un enchevêtrement impossible à rompre :

«De même que notre corps en son unité possède plus d'un membre et que ces membres n'ont pas tous la même fonction, ainsi nous, à plusieurs, nous ne formons qu'un seul corps dans le Christ, étant chacun pour sa part membres les uns des autres

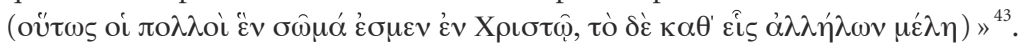

C'est surtout 1 Corinthiens qui semble un écho presque direct de la fable de Ménénius Agrippa, après une réaffirmation de l'unité du corps du $\mathrm{Christ}^{44}$ :

"L'œil ne peut donc dire à la main: "Je n'ai pas besoin de toi" ni la tête à son tour dire aux pieds: "Je n'ai pas besoin de vous" "

Il n'est ni nécessaire, ni possible d'évoquer ici le développement donné par les Pères de l'Église à ces considérations d'unité mystique et de solidarité absolue entre les membres du corps du Christ, différenciés par leur fonction, avec une différenciation qui s'avoue clairement comme une hiérarchie, dans laquelle les uns doivent commander et les autres se soumettre. Cette différenciation essentielle, plus d'une fois mise en lumière, apparaît comme parfaitement antinomique des idées d'Aristote

42. Paul, Ep. 1, 22 et Col. 1, 18 affirmant la primauté universelle du Christ, à compléter par son corollaire 1 Co. 6, 15. Traduction de la Bible de Jérusalem (1955), de même pour les extraits suivants.

43. Paul, Rm. 12, 4-5. La même expression est reprise en Ep. 4, 25, pour justifier la totale sincérité que se doivent les membres de la communauté.

44. Paul, 1 Co. 12, 12-14.

45. Paul, 1 Co. 12, 21. 
sur la successivité pour le citoyen de l'autorité $\alpha \rho \chi \varepsilon ı v$ et de l'obéissance ő $\rho \chi \varepsilon \sigma \theta \alpha$. Nous ne citerons qu'un exemple particulièrement significatif, chez Grégoire de Nazianze, qui n'est pourtant pas le plus autoritaire des Pères:

De même qu'il y a dans le corps un élément qui commande et qui est pour ainsi dire

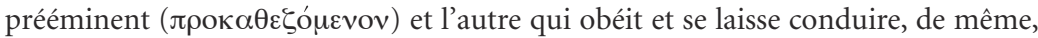
dans les Églises aussi, Dieu a établi, par une loi d'égalité qui touche au mérite ou à la sagesse par laquelle il a tout lié, que les uns soient le troupeau et obéissent, tous ceux à qui cela est plus avantageux, en se laissant diriger en paroles et en actes vers leur devoir, et que les autres soient leurs bergers et leurs maîtres d'école pour le bon ordre de l'Église ${ }^{46} \ldots$

Il est juste de signaler que la dimension mystique ( «membres les uns des autres» $\left.\dot{\alpha} \lambda \lambda \eta^{\prime} \lambda \omega \nu \mu \varepsilon^{\prime} \lambda \eta\right)$ est aussi soulignée dans les commentaires ${ }^{47}$ comme indissociable du caractère hiérarchique de la métaphore des membres du corps de l'Église, dont le Christ est la tête; ainsi dans cet autre passage du même Grégoire :

Un membre commande et dispose de la prééminence, l'autre obéit et se laisse diriger et l'activité des deux n'est pas identique, si du moins commander et obéir ne sont pas identiques; les deux deviennent un, pour contribuer à l'unité du Christ, en se laissant mettre en harmonie et ajuster par le même Esprit ${ }^{48}$.

\section{L'humanisme et la combinaison des deux traditions de la métaphore du corps}

Mais il est certain qu'à travers les siècles la métaphore chrétienne de l'Église comme corps, en raison de l'association de cette dernière avec les pouvoirs humains, a pris un tour nettement politique, clairement perçu par les humanistes. C'est ainsi que le rapport entre la fable paienne de Ménénius Agrippa et l'épître paulinienne avait déjà frappé les humanistes des XVI ${ }^{\mathrm{e}}$ et XVII ${ }^{\mathrm{e}}$ siècles, qu'il s'agisse de commentateurs religieux de l'épître, ou de penseurs politiques aux origines de la réflexion occidentale; c'est ce que met en lumière un commentateur allemand ${ }^{49}$ qui, après avoir étudié le champ des métaphores liées au pouvoir, s'est attaché à l'étude approfondie de la fable du conflit entre les membres et l'estomac. Ainsi rappelle-t-il que J. Bodin citait la Première Lettre aux Corinthiens pour se tourner contre le principe

46. Grégoire de Nazianze, Apologetica, $P G, 35,409,19-25$. Traduction personnelle ainsi que pour les deux passages suivants de Grégoire de Nazianze.

47. Ainsi par exemple dans Grégoire de Nazianze, De Pace, 1, PG, 35, 748, 1. 38, concernant les ménagements à garder et les condamnations à éviter pour préserver l'articulation des membres de la communauté.

48. Grégoire de Nazianze, De moderatione in disputando, $P G, 36,185,1.45$.

49. Peil 1985, 191-192, n. 500, évoque Calvin parmi d'autres commentateurs contemporains. 
d'égalité démocratique décrit par Aristote et comprenait la fable d'Agrippa comme un argument opposé à une égalité politique absolue ${ }^{50}$.

Nous avons personnellement relevé un indice intéressant du fait que les humanistes étaient eux-mêmes imprégnés de la métaphore du corps représentant la cité ou l'État au point qu'une fois, le mot « corps » a été ajouté à un texte ancien qui ne le comportait pas: dans la Vie de Solon de Plutarque les éditeurs modernes ${ }^{51}$ insèrent une conjecture de l'humaniste allemand Xylander, qui n'est nullement indispensable. Le biographe grec évoque une loi de Solon qui permettait à qui le voulait - et non à la victime seulement ou à ses ayants droit - de poursuivre en justice un coupable de violences et, à presque sept siècles de distance, il s'aventure à donner les motifs de cette loi, qu'il approuve:

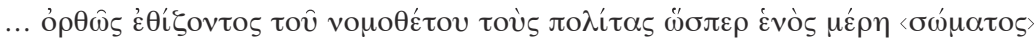

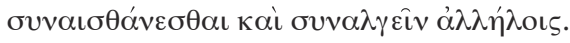

«Le législateur, avec raison, avait voulu par là accoutumer les citoyens à ressentir et partager, comme étant les parties d'un seul [corps], les maux les uns des autres $»^{52}$.

Il faudrait traduire plus abstraitement «comme étant les parties d'une seule réalité». Le terme $\sigma \hat{\omega} \mu \alpha$ n'est pas dans le texte. Or Plutarque occupe une position charnière dans notre étude : héritier de la tradition classique - ce qui pourrait expliquer l'absence de la métaphore dans ce passage, qui ne met en lumière que la solidarité et non la hiérarchie entre les composantes de la cité - il figure aussi parmi les témoins d'époque romaine pour la fable de Ménénius Agrippa et connaissait donc bien les valeurs, hostiles à la démocratie, véhiculées par cette métaphore.

\section{Bilan}

Ce survol beaucoup trop rapide d'une immense question amène à se demander comment la métaphore du corps de la cité, foncièrement aristocratique et inégalitaire dans ses origines archaïques, qu'elles soient grecques ou proche-orientales, a pu s'implanter à partir de la Renaissance jusqu'à devenir banale dans la réflexion politique moderne, marquée par l'évolution vers la démocratie.

À notre avis, l'image s'est d'abord banalisée à partir de son emploi religieux, puis elle a subi une usure et un dessèchement qui l'ont amenée à une totale abstraction et l'ont en quelque sorte "inactivée », au point que nous ne percevons aucune atteinte à notre autonomie personnelle si nous sommes désignés comme «membres »

50. Peil 1985, 192 : l'auteur cite la conclusion de Bodin : «J'ai bien voulu user de cet exemple, et monstrer au doigt et à l'œil les inconvénients qui suivent l'état populaire. »

51. Ziegler 1969 aussi bien que Flacelière 1961.

52. Plutarque, Vie de Solon (Flacelière 1961), 18, 6. 
du «corps » social ou politique, là où Aristote s'astreignait à parler de façon neutre et abstraite de «tout» et de "parties». C'est ce qui explique que les savants commentateurs d'Aristote que nous avons cités emploient couramment une métaphore absente de son œuvre.

Pourtant il nous semble important de bien souligner la distance qui sépare de l'unité mystique et hautement hiérarchisée sous-jacente à l'emploi chrétien de la métaphore la représentation aristotélicienne de la communauté des hommes

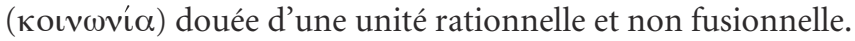

Michelle LACORE

Université de Caen Basse-Normandie

\section{Références bibliographiques}

Allan D.J. (1965), «Individual and State», in «Politique» d'Aristote, Genève, Fondation Hardt (Entretiens sur l'Antiquité classique ; 11), p. 55-85.

Aristote, Politica, W.D. Ross (éd.) (1957), Oxford, Clarendon Press.

Aristote, Politique 2 , livres I-VIII, J. Aubonnet (éd. et trad.) (1986-1991), Paris, Les Belles Lettres (CUF), tomes 1-3, 5 vol. avec introduction, notices, notes, index général et index verborum, traduction rééditée dans Aristote, Politique, Paris, Gallimard (Tel), 1993.

Aubenque P. (1993), «Aristote et la démocratie», in Aristote politique. Études sur la "Politique» d'Aristote, P. Aubenque, A. Tordesillas (dir.), Paris, PUF, p. 255-264.

BArker E. (1959), The political thought of Plato and Aristotle, New York, Dover Publications.

Canto-Sperber M. (1993), «L'unité de l'État et les conditions du bonheur public (Platon, République, V; Aristote, Politique, II) », in Aristote politique. Études sur la "Politique» d'Aristote, P. Aubenque, A. Tordesillas (dir.), Paris, PUF, p. 49-72.

Cassius Dio, Historiae Romanae (versio 1 in vol. 1), U.P. Boissevain (éd.) (1895, reimpr. 1955), Berlin, Weidmann, 46-47 = Zonaras, Epitome historiarum, lib. 1-12, L. Dindorf (éd.) (1869), vol. 2, lib. 6-10, Leipzig, Teubner, 7, 14, 6-14 (références du TLG-E).

Corpus fabularum Aesopicarum, A. Hausrath, H. Hunger (éd.) (1959), Leipzig, Teubner, 1.2, fab. 132, comprenant aussi Fabulae Syntipae philosophi, fab. 35.

Denys D’Halicarnasse, Antiquitates Romanae, K. Jacoby (éd.) (1885-1905), Leipzig, Teubner (repr. Stuttgart, 1967).

DodDs E.R. (1969) Gorgias, A Revised Text with Introduction and Commentary, Oxford, Clarendon Press. 
Grégoire de Nazianze, Orationes.

Grégoire de Nazianze, Apologetica, or. 2, PG, 35, p. 408-513.

Grégoire de Nazianze, De moderatione in disputando, or. 32, PG, 36, p. 173-212.

Grégoire de Nazianze, De Pace, or. 6, PG, 35, p. 721-752.

Hy péride, Discours, G. Colin (éd. et trad.) (1946), Paris, Les Belles Lettres (CUF).

Isocrate, Discours, vol. 3, Aréopagitique, G. Mathieu (éd. et trad.) (1942), Paris, Les Belles Lettres (CUF) ; vol. 4, Panathénaïque, G. Mathieu, É. Brémond (éd. et trad.) (1962), Paris, Les Belles Lettres (CUF).

Nouveau Testament, K. Aland, M. Black, C.M. Martini, B. M. Metzger, A. Wikgren (éd.) (1968), The Greek New Testament, $2^{\text {nd }}$ éd., Stuttgart, Würtemberger Bible Society, traduction sous la direction de l'École biblique de Jérusalem, Paris, Desclée de Brouwer, 1955.

Ogilvie R.M. (1965), A Commentary on Livy, Books 1-5, Oxford, Clarendon Press.

PeIL D. (1985), Der Streit der Glieder mit dem Magen, Francfort-sur-le-Main - Berne - New York, Peter Lang.

Perry B.E. (1952), Aesopica, Urbana, University of Illinois Press.

Platon, Gorgias, 464b, A. Croiset (éd. et trad.) (1923), Paris, Les Belles Lettres (CUF).

Platon, République, É. Chambry (éd. et trad.) (1932-1934), Paris, Les Belles Lettres (CUF).

Plutarque, Vies II, Vie de Solon, R. Flacelière (éd. et trad.) (1961), Paris, Les Belles Lettres (CUF).

Plutarque, Plutarchi vitae parallelae 4, K. Ziegler (éd.) (1969), Leipzig, Teubner, Solon, vol. 1, 1, p. 82-123.

Tite-Live, Histoire romaine, E. Lasserre (éd. et trad.) (1950), Paris, Garnier.

Weil R. (1960), Aristote et l'histoire: essai sur la «Politique», Paris, Klincksieck.

West M.L. (1969), «Near Eastern Material in Hellenistic and Roman Literature », HSCPh, 73 , p. 113-134.

Zafiropoulos C. (2001), Ethics in Aesop's Fables. The Augustana Collection, Leyde, Brill.

Zonaras, Epitome historiarum, lib. 1-12, L. Dindorf (éd.) (1869), vol. 2, lib. 6-10, Leipzig, Teubner, 7, 14, 6-14 (références du TLG-E). 